\title{
Errata
}

BERICHTIGUNG ZU "QUASIRATIONALE SINGULARITÄTEN"

\section{Axel Vogt}

Die Arbeit [1] enthält einen Fehlschluß, der zu der falschen Aussage 3.4.a führt. Die Definition einer quasirationalen Singularität läßt sich jedoch so modifizieren, daß zwar die Aussagen 3.1 bis 3.5 geeignet umformuliert werden müssen, die Hauptergebnisse 3.6 bis 3.8 jedoch unverändert bleiben.

DEFINITION. Ein normalex, reindimensionaler Raum $X$ mit Desingularisierung $\delta: Y \rightarrow X$ heiBe quasirational (in $X \in X$ ), falls (in $x$ ) die spur $\delta_{*} \omega_{Y} \rightarrow K_{X}$ bijektiv, also $\delta_{*} \omega_{Y}$ (in $X$ ) reflexiv ist; $X$ heiße k-quasirational (in $x$ ), falls zusätzlich $K_{X}$ (für eine offene Umgebung von $x$ ) die Bedingung $\left(S_{K+1}\right.$ ) erfullt.

Beispiel 2.4 und 2.6 gelten auch fur diese modifizierte Definition; die Aussage 2.3 ist zu streichen.

In Lemma 2.5 und dessen Beweis hat man stets die rektifizierte Tiefe prof* $(F)=\inf \left\{\operatorname{prof}\left(F_{X}\right): x \in X\right.$ abgeschlossener

Punkt\} zu verwenden.

[1] VOGT, A.: Quasirationale Singularitäten. manuscripta math. $57,239-247$ (1987)

Axel Vogt

NWF I

Universität Regensburg

D - 8400 Regensburg 